\title{
Moyamoya disease associated with arteriovenous malformation and anterior communicating artery aneurysm: A case report and literature review
}

\author{
JINLU YU $^{1}$, YONGJIE YUAN ${ }^{1}$, DUODUO ZHANG ${ }^{2}$ and $\mathrm{KAN} \mathrm{XU}^{1}$ \\ ${ }^{1}$ Department of Neurosurgery, First Hospital of Jilin University; ${ }^{2}$ Department of Radiotherapy, \\ Third Hospital of Jilin University, Changchun, Jilin 130021, P.R. China \\ Received February 2, 2015; Accepted April 5, 2016
}

DOI: $10.3892 /$ etm.2016.3289

\begin{abstract}
Moyamoya disease (MMD) can be associated with an aneurysm or arteriovenous malformation (AVM). However, no case of MMD simultaneously associated with both intracranial aneurysm and AVM has been previously reported. The present study reports the case of a patient with MMD simultaneously associated with both aneurysm and AVM. The patient was a 46-year-old woman presenting with a subarachnoid hemorrhage whose imaging diagnosis of MMD was associated with an aneurysm and AVM. The aneurysm was located in the anterior communicating artery, which was similar to a berry aneurysm caused by hemodynamics. The AVM was located in the posterior circulation. Beyond the presentation of the posterior cerebral artery, the appearance of an artery supplying blood from the middle cerebral artery supported the view that the AVM was congenital and unruptured. Conservative treatment was provided and examination of the patient at follow-up showed good recovery. In addition to the case report, the present study also reviewed the relevant literature in order to compile information on MMD associated with both an aneurysm and AVM.
\end{abstract}

\section{Introduction}

Moyamoya disease (MMD) refers to stenosis or occlusion at the end of the bilateral internal carotid arteries, with a representative characteristic of the disease being the formation of moyamoya-like vessels; this condition is common in Japan and China (1). Although the majority of MMD cases are not caused by aneurysms, its association with an intracranial aneurysm is not uncommon (2). The aneurysms are often located in the

Correspondence to: Dr Kan Xu, Department of Neurosurgery, First Hospital of Jilin University, 71 Xinmin Avenue, Changchun, Jilin 130021, P.R. China

E-mail: jlyu@jlu.edu.cn

Key words: moyamoya disease, anterior communicating artery aneurysm, arteriovenous malformation posterior circulation, which is most likely the cause of the intracranial hemorrhage (3). Compared with its association with aneurysms, MMD associated with arteriovenous malformation (AVM) is rare, with only $\sim 20$ cases having been reported to date (4). MMD simultaneously associated with both intracranial aneurysm and AVM is very rare, with no case having been reported to date. The present study reports a case of MMD simultaneously associated with both intracranial aneurysm and AVM, and the possible cause of the concomitant aneurysm and AVM was investigated.

\section{Case report}

A 46-year old female patient was admitted to the First Hospital of Jilin University (Changchun, China) of 1st May 2014 due to a sudden and severe headache with nausea and vomiting. The patient was healthy, with no prior history of hypertension or diabetes. A physical examination revealed that the patient was conscious and able to answer questions accurately, and that her limbs were flexible with normal muscle tone, muscle strength grade $\mathrm{V}$, a positive Kernig's sign, and a Hunt-Hess grade of III $(5,6)$.

Following admission, a head computed tomography (CT) scan (SOMATOM Definition AS; Siemens AG, Munich, Germany) revealed a high-density cord-like shadow in the suprasellar cistern, the ambient cistern and the lateral Sylvian cistern, and a high-density calcification in the left occipital lobe, whereas the bilateral ventricles were of normal size. Magnetic resonance imaging (MRI; MAGNETOM Trio, A Tim System; Siemens AG) showed a flow-void signal in the left occipital lobe, suggesting AVM (Fig. 1). In addition, MRI revealed a mass of tortuous vessels in the left occipital lobe, and the branch of the left posterior cerebral artery was observed passing into the lesion, with an observable bent and inappropriately shaped draining vein. The bilateral middle cerebral artery and the right anterior cerebral artery A1 segment were abnormally displayed, and an abnormal vascular network was observed in the area that the bilateral middle cerebral arteries supplied to, suggesting MMD; the left anterior cerebral artery was clearly observable, and a berry-like projection was observed at the anterior communicating artery (Fig. 2). CT angiography (CTA; SOMATOM Definition AS) showed 


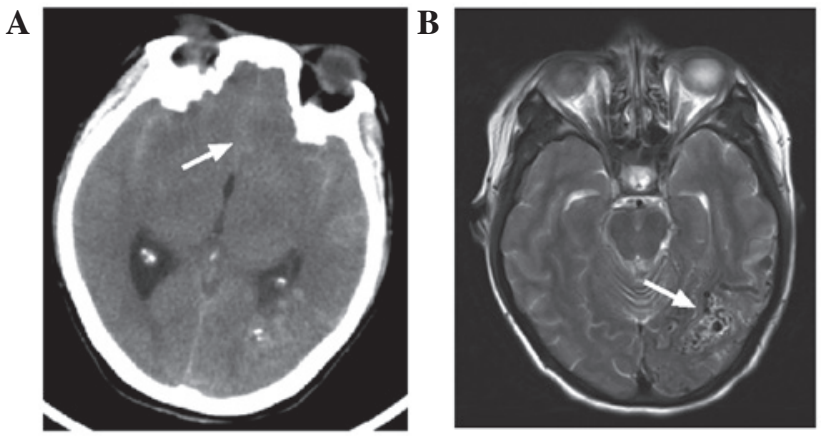

Figure 1. CT and MRI imaging of the head of the patient. (A) CT scan showed a high-density cord-like shadow in the lateral Sylvian cisterns and the longitudinal Sylvian (white arrow), suggesting a subarachnoid hemorrhage. (B) MRI scan revealed a flow-void signal in the left occipital lobe (white arrow), suggesting arteriovenous malformation. CT, computed tomography; MRI, magnetic resonance imaging.
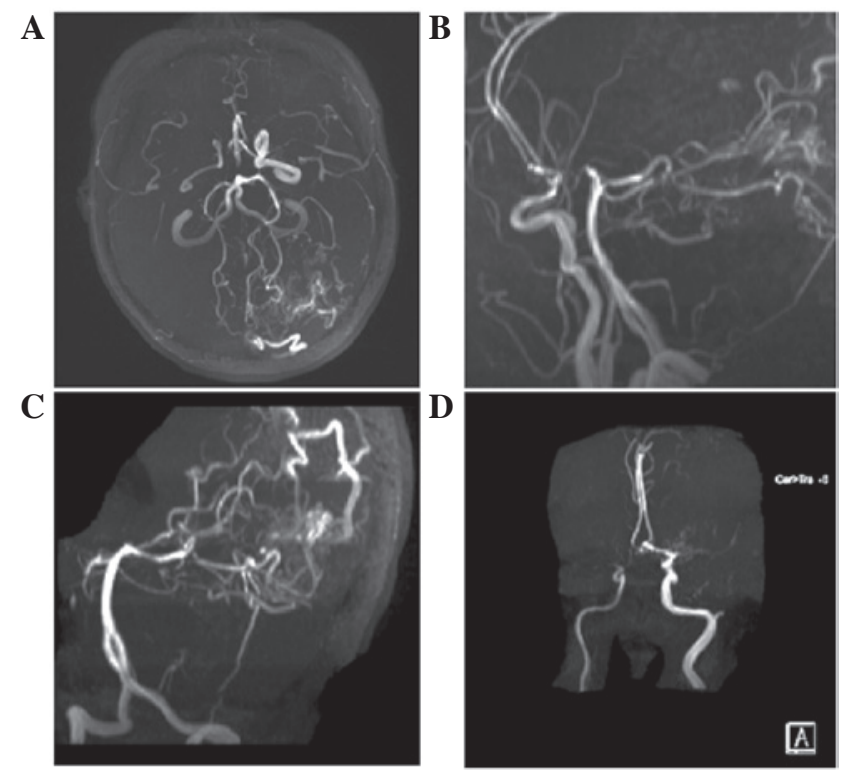

Figure 2. Magnetic resonance angiography of the head of the patient. (A-C) A mass of vessels was evident in the left occipital lobe and in the branch of the left posterior cerebral artery traveling into the lesion, with a bent and out-of-shape draining vein observed. The right carotid artery was narrower than the left. The bilateral middle cerebral artery and the right anterior cerebral artery A1 segment were not normally displayed, and a small abnormal vascular network was observed close to the bilateral middle cerebral artery, suggesting Moyamoya disease. (D) The left anterior cerebral artery was clearly observable, and a berry-like projection was present at the anterior communicating artery (white arrow), suggesting moyamoya disease, arteriovenous malformation and anterior communicating artery aneurysm.

the presence of an AVM in the blood supply near the left middle cerebral artery and the posterior cerebral artery, and the draining vein converged into the superior sagittal sinus upward and the transverse sinus backward. An aneurysm was observed in the anterior communicating artery, with a size of $\sim 4.6 \times 4.2 \mathrm{~mm}$, projecting to the lower right side (Fig. 3). A digital subtraction angiography (DSA; Siemens Neurostar Plus; Siemens AG) examination of the intracranial arteries was performed to confirm the diagnosis of MMD, the presence of AVM in the left occipital lobe, and the anterior communicating artery aneurysm (Fig. 4). Intracranial magnetic resonance perfusion imaging (MRP; MAGNETOM Trio, A Tim System)
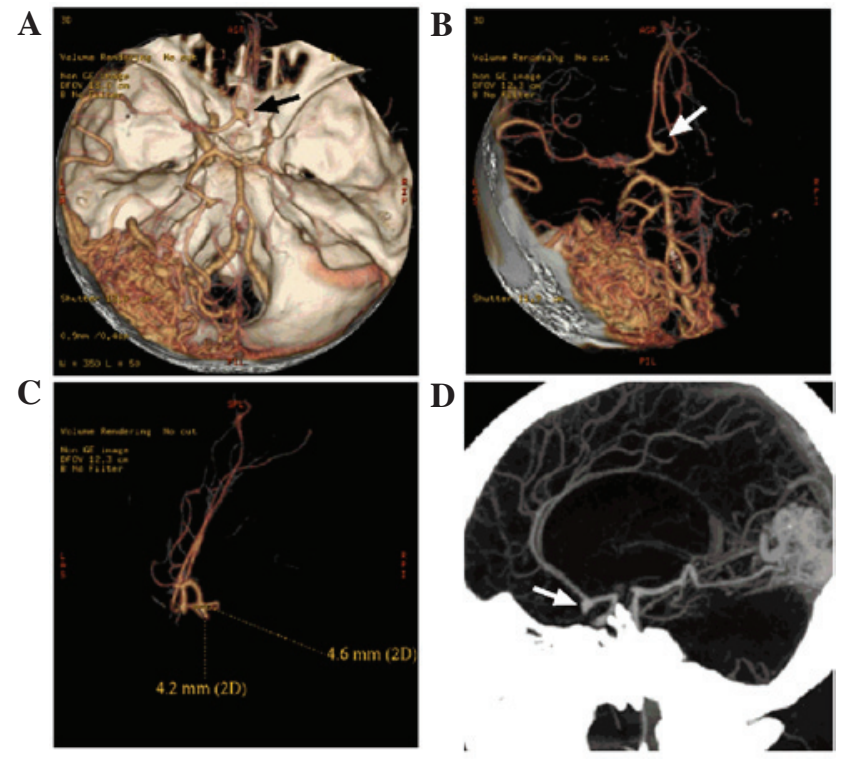

Figure 3. Computed tomography angiography image of the head of the patient. (A and B) An AVM was evident in the left occipital lobe. The branches of the left middle cerebral artery and posterior cerebral artery can be observed entering the lesion. The draining veins from the lesion converged into the superior sagittal sinus upward and the transverse sinus backward. The right middle cerebral artery and the anterior cerebral artery A1 segment were not normally displayed, and the left anterior cerebral artery A2 segment started from the anterior communicating artery (black and white arrows). A blood vessel branch in close to the left middle cerebral artery extended into the AVM. (C and D) An aneurysm of $\sim 4.6 \times 4.2 \mathrm{~mm}$ was observed in the anterior communicating artery (white arrows), projecting to the lower right side. AVM, arteriovenous malformation.

was performed, following the injection of gadolinium-DTPA (0.1 mmol/kg; Omniscan ${ }^{\mathrm{TM}}$; GE Healthcare Bio-Sciences, Pittsburgh, PA, USA) into the patient. Cerebral blood volume (CBV), cerebral blood flow (CBF), mean transit time (MTT) and time to peak (TTP) were measured by comparing the bilateral colors and time parameters; these results showed that the CBV, CBF, MTT and TTP were symmetrical in the bilateral hemispheres and that there were no significant ischemic changes in the brain tissue (Fig. 5).

Following admission, the patient underwent aneurysm-clipping surgery (7). Briefly, the sylvian fissure was dissected near the bifurcation of the carotid artery to expose the anterior cerebral artery A1 segment. Subsequently, the anterior communicating artery aneurysm was located and projected to the opposite side. The surgical clipping of the aneurysm was successful, with no intracranial hemorrhage or edema observed in the followed-up CT examination (Fig. 6A and B). The patient recovered well with no complications. At the follow-up CTA conducted six months following the surgical procedure neither an aneurysm recurrence nor any change in the MMD and AVM was observed (Fig. 6C-F). The present study was approved by the Ethics Committee of the First Hospital of Jilin University. Written informed consent was obtained from the patient for the publication of this study.

\section{Discussion}

The main characteristic of MMD is a stenosis or occlusion at the end of the internal carotid artery or a stenosis or occlusion at 

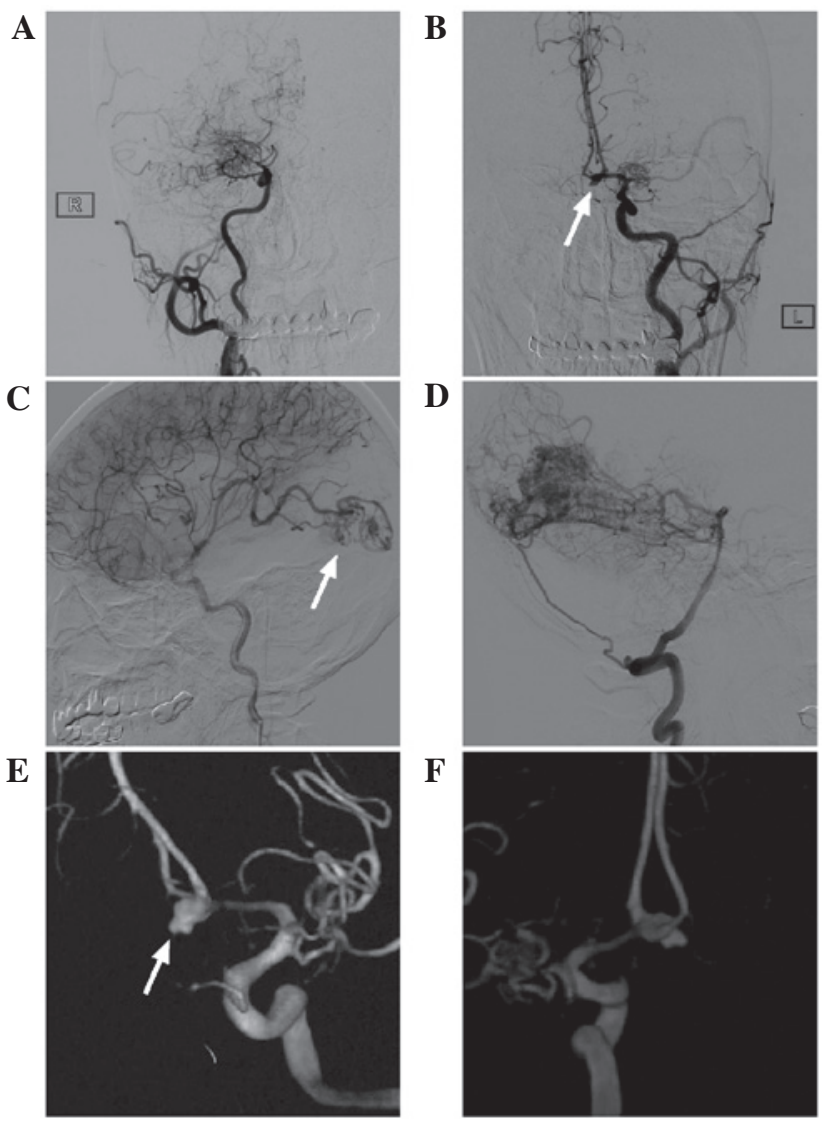

Figure 4. Digital subtraction angiography image of the head of the patient. (A and B) Carotid angiography showed that the bilateral middle cerebral artery was not present and was replaced by abnormally developed moyamoya-like vessels, and the right anterior cerebral artery was absent. The left anterior cerebral artery was dominant, serving the bilateral anterior cerebral artery, and an aneurysm was present in the anterior communicating artery (white arrow). (C and D) An arteriovenous malformation in the occipital lobe was observed, with the blood supply from the middle cerebral artery and the posterior cerebral artery (white arrow) and the draining veins converging into the superior sagittal sinus upward and the left transverse sinus backward ( $\mathrm{E}$ and $\mathrm{F}$ ) 3 dimensional reconstruction showed that the anterior communicating artery aneurysm had an irregular shape, with ruptured vesicles present on top (white arrow).

the end of the anterior cerebral artery and/or the middle cerebral artery; such a stenosis or occlusion results in the compensatory expansion of a moyamoya-like vessel network in the area adjacent to the stenosis (8). Following the occurrence of MMD, the pressure on the artery trunk with no occlusion increases, and an aneurysm may occur. This aneurysm is typically located in the posterior circulation, specifically at the tip of the basilar artery (9). If the end of the carotid artery is not entirely occluded following MMD, the anterior cerebral artery may be unobstructed, and a compensatory increase in blood flow will induce the formation of an aneurysm at the anterior communicating artery (2). In 1996, Kawaguchi et al (10) reported two cases of MMD associated with anterior communicating artery aneurysms and the image of the aneurysm reported in the present study is similar to that observed by Kawaguchi et al (10). Clipping surgery is a treatment option for MMD-associated anterior communicating artery aneurysms; however, intraoperative damage to the collateral circulation that forms following MMD should be avoided (11). The anterior communicating artery aneurysm reported in the present study was adequately
A

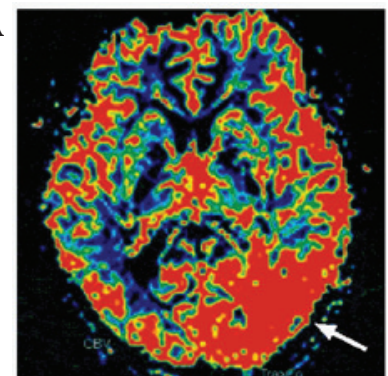

B

C

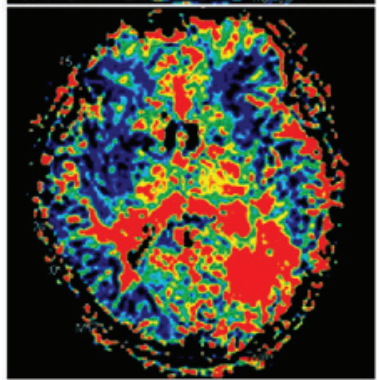

D

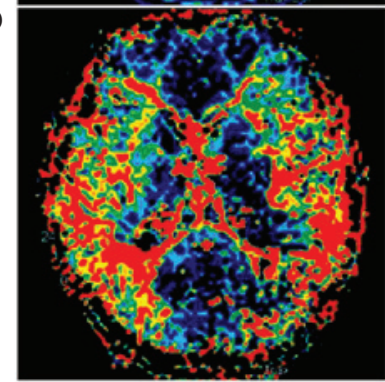

Figure 5. Magnetic resonance perfusion of the head of the patient. (A) Cerebral blood volume. (B) Cerebral blood flow. (C) Mean transit time. (D) Time to peak. No significant ischemic changes in the brain tissue were observed. The arrow indicates the location of the arteriovenous malformation.
A

C

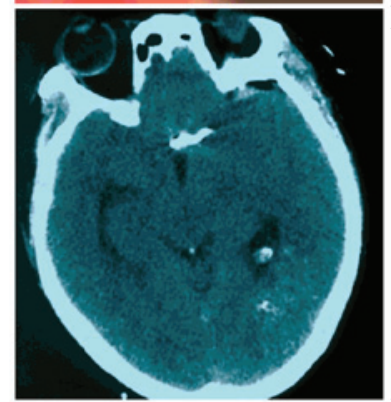

E

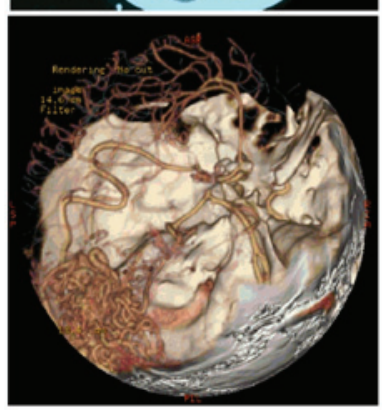

B

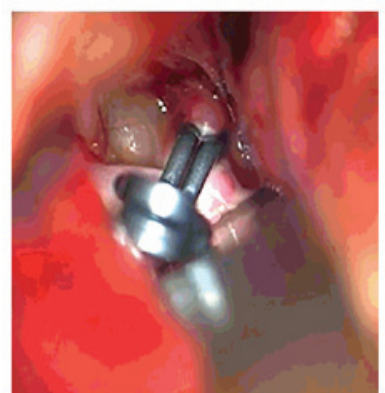

D

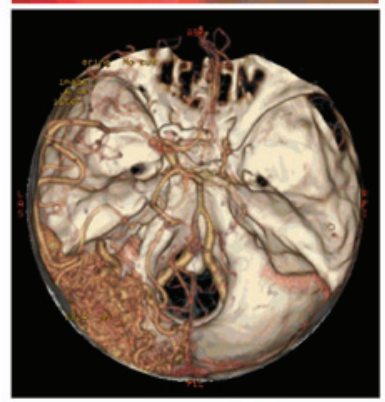

F

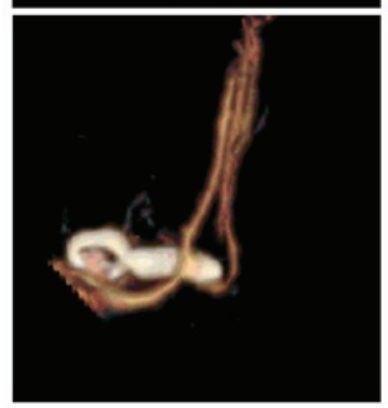

Figure 6. Intraoperative and postoperative images of the head of the patient. (A) A preoperative image of the anterior communicating aneurysm. (B) A postoperative image of the clipped anterior communicating aneurysm. (C) A postoperative computed tomography scan showed intact morphology of the brain tissue. (D-F) The follow-up computed tomography angiography six months after the surgical procedure showed a good result for the aneurysm clipping, with no changes in Moyamoya disease or arteriovenous malformation. 
evaluated prior to treatment. No obvious intracranial collateral circulation was observed in the area surrounding the surgical site following CTA or DSA, and the aneurysm-clipping surgical procedure resulted in improved prognosis. The present case of anterior communicating artery aneurysm showed a typical berry characteristic, which is in line with hemodynamic stress.

In rare cases, MMD has also been associated with AVM (12). Recent publications on the topic were reviewed and summarized in 2014 by Noh et al (4), who reported two cases and reviewed a further 17 cases of AVM-associated MMD. However, to the best of our knowledge, no case of MMD simultaneously associated with aneurysms and AVM has previously been reported. The pathogenesis of MMD associated with AVM has yet to be elucidated; however, it has been hypothesized that the occurrence of MMD may stimulate AVM. This hypothesis is predominantly based on the fact that MMD and AVM share numerous underlying pathogenic mechanisms, including the expression of vascular endothelial growth factors and inflammatory molecules. In addition, AVM is often located in the abnormally developed moyamoya-like vessels or in the compensatory collateral circulation $(4,13)$. Another hypothesis is that MMD and AVM are formed during congenital development, meaning that similar molecular mechanisms during embryonic development underlie the occurrence of MMD and AVM, and AVM has predilection sites similar to those for common intracranial AVMs (4). The retrospective analysis of AVM-associated MMD cases carried out by Noh et al (4) demonstrated that AVMs associated with MMD predominantly occur in the anterior circulation. The AVM associated with MMD in the present case report was located in the posterior circulation, with a blood supply provided predominantly by the posterior cerebral artery, which does not differ from common AVM (14). However, a unique characteristic of the $\mathrm{AVM}$ reported in the present study was a branch of the left middle cerebral artery extending backward to supply the AVM. Due to the fact that the AVM was large and located in the distribution area of the middle cerebral artery, it did not have the morphology of the newly-generated blood vessels characteristic of MMD. Therefore, the AVM-associated case of MMD in the present report supports the congenital development as the correct hypothesis.

For cases of MMD associated with both aneurysm and AVM, providing an appropriate treatment depends on the clinical manifestations of the patient. For an aneurysm associated with MMD, if the aneurysm has ruptured or is at high risk for rupture, the aneurysm should be treated with high priority to prevent rupture and hemorrhage (15). In addition to the treatment of the aneurysm, the treatment of MMD must be considered. For instance, if the MMD manifests as ischemia, a revascularization treatment is required (16). For the case reported in the present study, the anterior communicating artery aneurysm ruptured and, in order to prevent re-rupture, the aneurysm was clipped. The postoperative follow-up CTA showed that re-rupture had not occurred. Since the patient with MMD reported in the present study had no history of cerebral ischemia, and the MRI of the patient showed no infarction, ischemia was not suspected. Furthermore, MRP showed the absence of marked brain tissue ischemia, and therefore no treatment was provided for MMD. No collateral circulation associated with MMD was observed at the surgical site, as determined by DSA, and therefore a craniotomy was conducted. For AVM associated with MMD, the treatment strategy is similar to that recommended for a conventional case. The majority of AVMs are only occasionally discovered, and treatment is not required (17). Among the two cases reported by Noh et al (4) and the 17 cases summarized in their study, only three cases were treated: Two patients received resections, and one patient underwent $\gamma$-knife radiation therapy (16). In the case of an AVM associated with MMD, treatment must be carefully undertaken. As MMD is usually accompanied by a wide range of collateral circulation issues, either craniotomy or interventional treatment must avoid damage or interference to the collateral circulation, in order to prevent the onset of symptoms (18). In this case report, the AVM was located in the posterior circulation with no hemorrhage, and the drainage was unobstructed; therefore, no treatment was provided. Currently, if no obvious risk factor for hemorrhage is observed in the case of unruptured AVM, follow-up observation is recommended (19).

The MMD case presented in the present report was simultaneously associated with both intracranial aneurysm and AVM. The aneurysm was located in the anterior communicating artery, which is similar to a common berry aneurysm caused by hemodynamic stress. The AVM was located in the posterior circulation. In addition to the posterior cerebral artery, there was one abnormal feeding artery supplying the AVM from the middle cerebral artery, which indicated that the AVM was congenital and unruptured. Therefore, conservative treatment was performed.

\section{References}

1. Hayashi K, Horie N, Izumo T and Nagata I: A nationwide survey on unilateral moyamoya disease in Japan. Clin Neurol Neurosurg 124: 1-5, 2014.

2. Zhang L, Xu K, Zhang Y, Wang X and Yu J: Treatment strategies for aneurysms associated with moyamoya disease. Int J Med Sci 12: 234-242, 2015.

3. Yeon JY, Kim JS and Hong SC: Incidental major artery aneurysms in patients with non-hemorrhagic moyamoya disease. Acta Neurochir (Wien) 153: 1263-1270, 2011.

4. Noh JH, Yeon JY, Park JH and Shin HJ: Cerebral arteriovenous malformation associated with moyamoya disease. J Korean Neurosurg Soc 56: 356-360, 2014.

5. Wartenberg KE: Critical care of poor-grade subarachnoid hemorrhage. Curr Opin Crit Care 17: 85-93, 2011.

6. Seeder L: Muscle strength grading. Ann Emerg Med 12: 407, 1983.

7. Yu J, Xu B, Guo Y and Xu K: Direct clipping of a blister-like aneurysm in the supraclinoid segment of the internal carotid artery: a clinical analysis of nine cases. Int J Clin Exp Med 8: 21786-21795, 2015.

8. Research Committee on the Pathology and Treatment of Spontaneous Occlusion of the Circle of Willis; Health Labour Sciences Research Grant for Research on Measures for Infractable Diseases: Guidelines for diagnosis and treatment of moyamoya disease (spontaneous occlusion of the circle of Willis). Neurol Med Chir (Tokyo) 52: 245-266, 2012.

9. Yu JL, Wang HL, Xu K, Li Y and Luo Q: Endovascular treatment of intracranial aneurysms associated with moyamoya disease or moyamoya syndrome. Interv Neuroradiol 16: 240-248, 2010.

10. Kawaguchi S, Sakaki T, Morimoto T, Kakizaki T and Kamada K: Characteristics of intracranial aneurysms associated with moyamoya disease. A review of 111 cases. Acta Neurochir (Wien) 138: 1287-1294, 1996.

11. Eom KS, Kim DW and Kang SD: Intracerebral hemorrhage caused by rupture of a giant aneurysm complicating superficial temporal artery-middle cerebral artery anastomosis for moyamoya disease. Acta Neurochir (Wien) 152: 1069-1073, 2010. 
12. Wu TC, Guo WY, Wu HM, Chang FC, Shiau CY and Chung WY: The rare association of moyamoya disease and cerebral arteriovenous malformations: A case report. Korean J Radiol 9 Suppl: S65-S67, 2008

13. Fujimura M, Kimura N, Ezura M, Niizuma K, Uenohara $H$ and Tominaga T: Development of a de novo arteriovenous malformation after bilateral revascularization surgery in a child with moyamoya disease. J Neurosurg Pediatr 13: 647-649, 2014.

14. Crowley RW, Ducruet AF, McDougall CG and Albuquerque FC: Endovascular advances for brain arteriovenous malformations. Neurosurgery 74 (Suppl 1): S74-S82, 2014.

15. Yang S, Yu JL, Wang HL, Wang B and Luo Q: Endovascular embolization of distal anterior choroidal artery aneurysms associated with moyamoya disease. A report of two cases and a literature review. Interv Neuroradiol 16: 433-441, 2010.
16. Iwama $\mathrm{T}$, Todaka $\mathrm{T}$ and Hashimoto $\mathrm{N}$ : Direct surgery for major artery aneurysm associated with moyamoya disease. Clin Neurol Neurosurg 99 (Suppl 2): S191-S193, 1997.

17. Nerva JD, Mantovani A, Barber J, Kim LJ, Rockhill JK, Hallam DK, Ghodke BV and Sekhar LN: Treatment outcomes of unruptured arteriovenous malformations with a subgroup analysis of ARUBA (A Randomized Trial of Unruptured Brain Arteriovenous Malformations)-eligible patients. Neurosurgery 76: 563-570; discussion 570; quiz 570, 2015.

18. Somasundaram S, Thamburaj K, Burathoki S and Gupta AK: Moyamoya disease with cerebral arteriovenous malformation presenting as primary subarachnoid hemorrhage. J Neuroimaging 17: 251-254, 2007.

19. Novakovic RL, Lazzaro MA, Castonguay AC and Zaidat OO: The diagnosis and management of brain arteriovenous malformations. Neurol Clin 31: 749-763, 2013. 